\title{
Opto-Electronic Properties of Fluorene-Based Derivatives as Precursors for Light-Emitting Diodes
}

\author{
Silvia Fratiloiu, ${ }^{\dagger}$ Sofia M. Fonseca, ${ }^{\ddagger}$ Ferdinand C. Grozema,,$\dagger$ Hugh D. Burrows, \\ Maria L. Costa, ${ }^{\S}$ Ana Charas," Jorge Morgado," and Laurens D. A. Siebbeles ${ }^{\dagger}$ \\ Opto-Electronic Materials Section, DelftChemTech, Delft University of Technology, Julianalaan 136, \\ 2628 BL Delft, The Netherlands, Departamento de Química, Universidade de Coimbra, 3004-535 Coimbra, \\ Portugal, Departamento de Matemática, Universidade de Aveiro, 3810-193 Aveiro, Portugal, and Instituto de \\ Telecomunicações, Instituto Superior Técnico, Avenida Rovisco Pais, P-1049-001 Lisboa, Portugal
}

Received: November 29, 2006; In Final Form: February 13, 2007

This paper reports optical absorption spectra of oxidized fluorene copolymers obtained by chemical oxidation with $\mathrm{Ce}(\mathrm{IV})$ and by pulse radiolysis experiments in chlorinated solvents. Comparison of the results observed by the two techniques is used to provide spectral data on the copolymer radical ions and information on stability of the oxidized species. In addition, a detailed quantum chemical characterization is presented, concerning the electronic and optical properties of three series of charged oligomers containing alternating fluorene and phenylene or thienylene or benzothiadiazole units, respectively. The introduction of the comonomer strongly influences the optical properties, leading to a red shift in the absorption spectra of the charged oligomers. This shift is more pronounced in the case of fluorene benzothiadiazole anions due to the strong electron-accepting character of the benzothiadiazole moieties. The charge distribution of the fluorene benzothiadiazole anion is different from that corresponding to fluorene phenylene and fluorene thienylene anions. The negative charge of the latter oligomers is evenly distributed over the fluorene units, while the former oligomer localizes the negative charge on the benzothiadiazole units. The charge distribution correlates with the optical absorption spectra. When the positive charge is localized on a different unit than the negative charge, the cation and anion spectra are different. Similar spectra are obtained if both the positive and negative charges are localized on the same unit.

\section{Introduction}

The development of tunable and flexible light-emitting diodes (LEDs) from conjugated polymers has received considerable attention in the last years. Fluorene oligomers and polymers are highly fluorescent compounds, ${ }^{1}$ which makes them suitable for applications in LEDs. They have good stability due to the rigidly planar biphenyl structure in the fluorene unit. The introduction of substituents at the C-9 position makes fluorene soluble, and thus easy to process from organic solvents. One disadvantage, which limits the use of polyfluorenes in bluelight-emitting diodes, is the difficulty to inject charges in these materials. To improve the charge injection, different electrondonating or accepting units have been incorporated into the structure of polyfluorenes. In this way, the energies of the highest occupied molecular orbital (HOMO) and the lowest unoccupied molecular orbital (LUMO) can be varied, making it easier to inject charges. Using quantum chemical calculations, Yang et $\mathrm{al}^{2}$ have shown that the introduction of the electrondonating 3,4-ethylenedioxythiophene units in the backbone increases the HOMO energy, which greatly improves the hole injection. Alternatively, the presence of 1,3,4-oxadiazole moieties leads to a lowering of the LUMO level and thus to an improvement of the electron-accepting properties. ${ }^{2}$

* Corresponding author. E-mail: f.c.grozema@tnw.tudelft.nl. Tel.: +3115-278 3914. Fax: +31-15-278 7421.

Delft University of Technology.

$\div$ Universidade de Coimbra.

$\S$ Universidade de Aveiro.

"Instituto Superior Técnico.
Structural, electronic, and optical properties of fluorene copolymers containing phenylene, ${ }^{3-6}$ thienylene, ${ }^{3,5,7-9}$ and benzothiadiazole ${ }^{10-12}$ moieties have been investigated experimentally and theoretically. Particular attention has been paid to the application of these copolymers in LEDs. Donat-Bouillud et al. ${ }^{13}$ have shown that the electroluminescent properties can be changed to a large extent by incorporating phenylene or thienylene moieties in the fluorene backbone. The spectral emission varies from blue to green or yellow, depending on the composition of the copolymers. Beaupre et al. ${ }^{14}$ have synthesized a fluorene-thiophene copolymer, which is promising for applications in orange and red-light-emitting diodes. A bright red polymer light-emitting device was fabricated by Kim et al. ${ }^{15}$ with blends of regioregular poly(3-hexylthiophene) and poly (9,9-dioctylfluorene-co-benzothiadiazole) (F8BT) as the emissive layer.

To improve the understanding of the experimental data, quantum chemical calculations have been performed at different levels of theory. Ab initio or density functional-based methods have been used to calculate structural properties, ionization potentials, electron affinities, energy gaps, and optical absorption spectra of fluorene-based co-oligomers. ${ }^{2-9,12}$ No experimental or theoretical work has been reported yet on optical absorption of charged fluorene co-oligomers and copolymers. Therefore, this study aims to investigate these properties for a better understanding of the effect of introducing different moieties on the fluorene backbone.

Chemical oxidation and reduction have been used to study the charged species of a variety of conjugated polymers and 


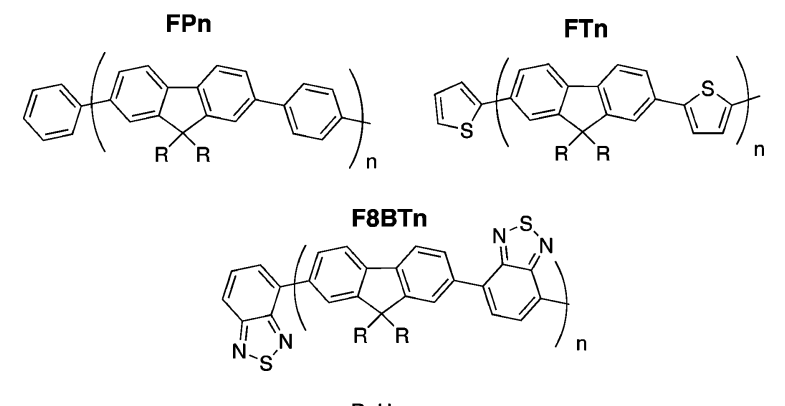

$\mathrm{R}=\mathrm{H}$

Figure 1. Chemical structure of the fluorene-based co-oligomers studied ( $n$ is the number of repeat units).

oligomers. ${ }^{16-21}$ One-electron oxidized (radical cation) or reduced (radical anion) species are produced initially. However, it has been shown that multistep oxidation and reduction reactions can occur; ${ }^{22-24}$ in addition, the initial product can undergo degradation processes ${ }^{25-27}$ such that the first species detected by conventional techniques may not be the one-electron oxidized or reduced charge carrier species. In contrast, pulse radiolysis has been shown to be an excellent technique for characterizing the initially formed charge carriers produced on one-electron oxidation or reduction. ${ }^{28,29}$ Comparison of behavior of the species prepared by the two techniques provides valuable information on their reactivity, which may be relevant to what happens in devices, and, in particular, to their long-term stability.

The present work involves experimental and theoretical studies of the optical properties of charged fluorene-based alternating copolymers and co-oligomers. The optical spectra of cations of poly[2,7-(9,9-bis(2'-ethylhexyl)fluorene)-alt-1,4phenylene] (PFP), poly[2,7-(9,9-bis(2'-ethylhexyl)fluorene)-alt2,5-thiophene] (PFT) and poly(9,9-dioctylfluorene-co-benzothiadiazole) (F8BT) were obtained by using chemical oxidation and pulse radiolysis techniques. To examine to what extent the nature of charges in fluorene co-oligomers is influenced by adding electron-donating or accepting units to the backbone, the absorption spectra of charged fluorene phenylene (FPn), fluorene thienylene (FTn), and fluorene benzothiadiazole (F8BTn) oligomers have been studied theoretically. The chemical structures of these oligomers are given in Figure 1. The results are compared with the absorption spectra of the corresponding charged fluorene (Fn) oligomers. ${ }^{30}$ The spectra of charged fluorene-based co-oligomers also provide valuable information about the spatial extent of charges along the chain. A detailed discussion about the distribution of an excess positive and negative charge along the fluorene-based co-oligomers chains is presented. The opto-electronic properties of the fluorene-based co-oligomers are studied as a function of chain length. This makes it possible to extrapolate the data from co-oligomers to copolymers and to compare the theoretical results with the experimental data.

\section{Experimental Details}

Details of the synthesis and photophysical properties of the three copolymers used in this study have been given elsewhere. ${ }^{31-33}$ These are all alternating copolymers, and details on molecular weights and polydispersities are given in ref 33. The fluorene copolymers were dissolved in carbon tetrachloride (with concentrations less than $10^{-4} \mathrm{M}$ in terms of repeat unit). Two techniques were tested to prepare the one-electron oxidized species. In the first, the copolymers in carbon tetrachloride solution were subjected to chemical oxidation, adding small quantities (ca $1 \%$ by volume) of a concentrated solution (50
$\mathrm{mM}$ ) of the strong oxidant cerium(IV) ammonium nitrate in acetonitrile (or $\mathrm{CD}_{3} \mathrm{CN}$ for the near-infrared (IR) region). The small amount of acetonitrile is not expected to change the properties of the oxidized species, and this has previously been shown to be an excellent technique for the quantitative preparation of the radical cations in chlorocarbon solvents of a variety of aromatic compounds. ${ }^{34,35} \mathrm{UV}-$ vis absorption spectra were measured on solutions in $1 \mathrm{~cm}$ quartz cuvettes on Shimadzu UV-2100 or Ocean Optics spectrophotometers. For the nearIR region $(\lambda>850 \mathrm{~nm})$, a Cary 14 Olis modified spectrometer was used. However, these chemical oxidation studies permit the observation of the radical cations only for a few seconds after they have been formed, and during this time they may undergo secondary reactions. We have, therefore, also used pulse radiolysis technique to prepare and investigate the cations of fluorene copolymers in carbon tetrachloride solutions. The pulse radiolysis experiments were carried out at the Free Radical Research Facility, Daresbury, UK. High-energy electron pulses with duration of $200 \mathrm{~ns}$ up to $2 \mu$ s were sent from a $12 \mathrm{MeV}$ linear accelerator to the copolymer solutions. The copolymer solutions were placed in a $2.5 \mathrm{~cm}$ optical path length quartz cuvette attached to a flow system. All solutions were bubbled with argon for about $30 \mathrm{~min}$ before experiments. The experimental setup and radiation chemistry of these solvents has been described in detail elsewhere. ${ }^{36}$

From the chemical oxidation experiments, the molar absorption coefficients for the oxidized species were calculated from the slope of the plot of the copolymer absorption values (at the maximum wavelength of absorption corrected for the baseline) versus the $\mathrm{Ce}(\mathrm{IV})$ concentration values, assuming $100 \%$ efficiency in the oxidation process. The limitations of this assumption will be discussed in Results and Discussion. In pulse radiolysis experiments, molar absorption coefficients were determined as functions of dose from the initial absorbance using tri( $p$-tolyl)amine $(10 \mathrm{mM})$ in carbon tetrachloride as dosimeter. ${ }^{34}$

\section{Computational Methodology}

The geometries of the three different series of fluorene oligomers were optimized using the Amsterdam density functional theory program. ${ }^{37}$ The geometry optimizations were performed using the local density approximation with exchange and correlation functionals based on Vosko-Wilk-Nusair parametrization of electron gas data. ${ }^{38}$ The generalized gradient approximation $^{39}$ corrections by Becke $^{40}$ (exchange) and Perdew $^{41}$ (correlation) were included. For optimizing the geometries, a double- $\zeta$ plus polarization basis set was used.

The electronic absorption spectra of the charged fluorene oligomers were calculated with time-dependent density functional theory (TDDFT), ${ }^{42,43}$ as implemented in the Q-Chem program. ${ }^{44}$ The excitation energies were computed using a correlation consistent ${ }^{45}$-polarized valence double zeta basis set. The Becke (exchange) and the Lee-Yang-Parr (correlation) ${ }^{46}$ functional (BLYP) was used.

The distribution of an excess positive and negative charge was calculated using density functional theory (DFT). The charge distribution was obtained from a Mulliken population analysis performed on the same charge density used to calculate the absorption spectra.

\section{Results and Discussion}

4.1. Experimental Optical Absorption Spectra of OneElectron Oxidized Species. As indicated in the experimental section, copolymer radical cations were prepared initially by chemical oxidation, using cerium(IV) ammonium nitrate as one- 
TABLE 1: Absorption Maxima and Molar Absorption Coefficients of the One-Electron Oxidized Copolymers Determined from Pulse Radiolysis and Chemical Oxidation ${ }^{a}$

\begin{tabular}{|c|c|c|c|c|c|c|}
\hline \multirow[b]{2}{*}{ copolymer } & \multicolumn{3}{|c|}{ pulse radiolysis } & \multicolumn{3}{|c|}{ chemical oxidation } \\
\hline & $\begin{array}{c}\lambda_{\max } \\
(\mathrm{nm})^{b}\end{array}$ & $\begin{array}{c}\epsilon \\
\left(\mathrm{M}^{-1} \mathrm{~cm}^{-1}\right)\end{array}$ & $\begin{array}{c}k \\
\left(s^{-1}\right)\end{array}$ & $\begin{array}{c}\lambda_{\max } \\
(\mathrm{nm})^{b}\end{array}$ & $\begin{array}{c}\epsilon \\
\left(\mathrm{M}^{-1} \mathrm{~cm}^{-1}\right)\end{array}$ & $\begin{array}{c}k \\
\left(\mathrm{M}^{-1} \mathrm{~s}^{-1}\right)\end{array}$ \\
\hline PFP & $540(2.30)$ & 2600 & $1.80 \times 10^{5}$ & $480(2.58)$ & 555 & see text discussion \\
\hline PFT & $680(1.82)$ & 5130 & $3.99 \times 10^{3}$ & $620(2.00)$ & 255 & 36.3 \\
\hline \multirow[t]{2}{*}{ F8BT } & $510(2.43)$ & 2460 & $7.10 \times 10^{3}$ & $680(1.82)$ & 510 & 9.1 \\
\hline & $720(1.72)$ & 1090 & $6.89 \times 10^{3}$ & & & \\
\hline
\end{tabular}

${ }^{a}$ The rate constants for the kinetics of the decay of the radical cations determined from pulse radiolysis and obtained from chemical oxidation experiments are also presented. ${ }^{b}$ Values in $\mathrm{eV}$ given in brackets.

electron oxidant. Figure 2 shows the absorption spectra of the species observed upon chemical oxidation of carbon tetrachloride solutions of the three fluorene copolymers upon increasing the $\mathrm{Ce}(\mathrm{IV})$ concentration. These all show a visible absorption band in the 500-700 nm region, accompanied by a longer wavelength feature. For example, oxidation of the fluorene phenylene copolymer (PFP) with $\mathrm{Ce}(\mathrm{IV})$ induces an absorption band at $480 \mathrm{~nm}$ through the proposed reaction

$$
\mathrm{Ce}(\mathrm{IV})+\mathrm{PFP} \rightarrow \mathrm{Ce}(\mathrm{III})+\mathrm{PFP}^{\bullet+}
$$

This absorption becomes more intense upon increasing the amount of $\mathrm{Ce}(\mathrm{IV})$. Good evidence for one-electron oxidation by this species has previously been presented for a number of systems, including aromatic amines, ${ }^{34,35}$ and the absorption is tentatively assigned to the one-electron oxidized species (positive charge). In support of this, the positively charged species is expected to show two absorption bands; ${ }^{47}$ the long wavelength feature may be attributed to the lowest energy transition of this species. The replacement of the phenyl ring with a thiophene ring or with a benzothiadiazole unit leads to a considerable red shift of the absorption maximum to 620 and $680 \mathrm{~nm}$, respectively. Molar absorption coefficients of the oxidized species of the three copolymers investigated in carbon tetrachloride have been calculated as described in Section 2, and their values and absorption maxima are listed in Table 1. It should be noted that although plots of absorption against [Ce(IV)] are relatively linear at low oxidant concentrations, they tend to plateau at higher values, possibly due to the fact that the copolymers have achieved their highest oxidation level.

For the PFT and F8BT copolymers, the oxidized species decay over several minutes, following pseudo-first-order kinetics. The decay of the PFT cation is shown in the Supporting Information (Figure S1a). The exponential fit of the decay of this transient gives a lifetime of $35.28 \mathrm{~s}$ for an added Ce(IV) concentration of $0.74 \mathrm{mM}$. This corresponds to pseudo-firstorder rate constant $\left(k_{\mathrm{obs}}\right)$ of $2.84 \times 10^{-2} \mathrm{~s}^{-1}$. The pseudo-firstorder rate constants $\left(k_{\text {obs }}\right)$ increase as a function of the added $\mathrm{Ce}(\mathrm{IV})$ concentration (see Supporting Information, Figure 1b). However, according to reaction 1, all the $\mathrm{Ce}(\mathrm{IV})$ has been quantitatively converted to $\mathrm{Ce}(\mathrm{III})$. The trend, therefore, indicates some more complex decay sequence, possible involving multiple charges on the chain. An empirical fit of the overall kinetic behavior of PFT and F8BT was made to the second-order rate law

$$
i=k[\mathrm{Ce}(\mathrm{IV})][\mathrm{X}]=k_{\mathrm{obs}}[\mathrm{X}]
$$

where [Ce(IV)] is the added cerium(IV) concentration, [X] represents the oxidized polymer species, and $k$ is the apparent second-order rate constant obtained from a plot of $k_{\text {obs }}$ against $\mathrm{Ce}(\mathrm{IV})$ concentration. Values of $k$ for the two copolymers are given in Table 1. For PFP, a somewhat different behavior was observed. At low $\mathrm{Ce}(\mathrm{IV})$ concentration $\left(4.95 \times 10^{-4} \mathrm{M}\right)$, the decay was pseudo-first-order with the rate constant $k=2.90 \times$ $10^{-3} \mathrm{~s}^{-1}$. Increase of the $\mathrm{Ce}(\mathrm{IV})$ concentration leads to a change of the kinetics, such that at $[\mathrm{Ce}(\mathrm{IV})]=1.22 \times 10^{-3} \mathrm{M}$, the decay could be fitted by a double exponential with fitting constants $k_{1}=7.23 \times 10^{-3} \mathrm{~s}^{-1}$ and $\mathrm{k}_{2}=2.94 \times 10^{-4} \mathrm{~s}^{-1}$.

One-electron oxidation of the polymers may be accompanied by side reactions, such as chain scission, leading to polymer degradation. ${ }^{25-27}$ In some cases, it is difficult to distinguish between the absorption spectrum of the initial positive charge carrier and of a side product. We have therefore carried out pulse radiolysis experiments, which allow direct observation of the initially formed positive charge carrier (radical cation). Radiolysis of chlorinated aliphatic solvents is known to quantitatively produce solvent radical cations, and using pulse radiolysis under these conditions, the copolymer radical cations $\left(\mathrm{S}^{+}\right)$are selectively generated, ${ }^{29,34,36}$ according to the reaction scheme (given for carbon tetrachloride):

$$
\begin{gathered}
2 \mathrm{CCl}_{4} \rightarrow \mathrm{CCl}_{4}^{\bullet+}+{ }^{\bullet} \mathrm{CCl}_{3}+\mathrm{Cl}^{-} \\
\mathrm{CCl}_{4}^{\bullet+}+\mathrm{S} \rightarrow \mathrm{CCl}_{4}+\mathrm{S}^{\bullet+}
\end{gathered}
$$

The optical absorption spectra of copolymer cations obtained by pulse radiolysis are presented in Figure 3, while spectral data of the species produced on pulse radiolysis and chemical oxidation are compared in Table 1.

Clear differences are seen in the spectral behavior of the species formed by pulse radiolysis and chemical oxidation. For all three copolymers, the visible absorption band seen in pulse radiolysis is red-shifted by $0.1-0.3 \mathrm{eV}$ relative to that seen by chemical oxidation. In addition, the molar absorption coefficients obtained by pulse radiolysis are at least five times greater than those obtained by chemical oxidation. Two factors may be responsible for this. Under the conditions of the pulse radiolysis experiments, the concentrations of solvent radical cation produced are lower than those of the copolymers, such that each polymer chain can only contain at most one positive charge. In contrast, from the concentrations (in $\mathrm{mg} / \mathrm{mL}$ ) and the average molecular weights of the copolymers, ${ }^{33}$ their molar concentrations are estimated to be less than $10^{-6} \mathrm{M}$ (based on the polymer chain), which is considerably lower than the $\mathrm{Ce}(\mathrm{IV})$ concentration. Under these conditions, it is possible to have more than one positive charge on each copolymer chain, either as doubly charged species (bipolaron) or as more than one relatively localized singly charged species on each polymer chain. These situations lead to different spectral signatures with the bipolarons predicted to give a single absorption band, while as indicated above single charges show two sub-band gap transitions. ${ }^{47}$ Pulse radiolysis of fluorene polymers using time-resolved microwave conductivity detection has shown that positive charges on fluorene-based polymers have very high mobility, ${ }^{48}$ and if two charges are formed on the same chain by oxidation with 

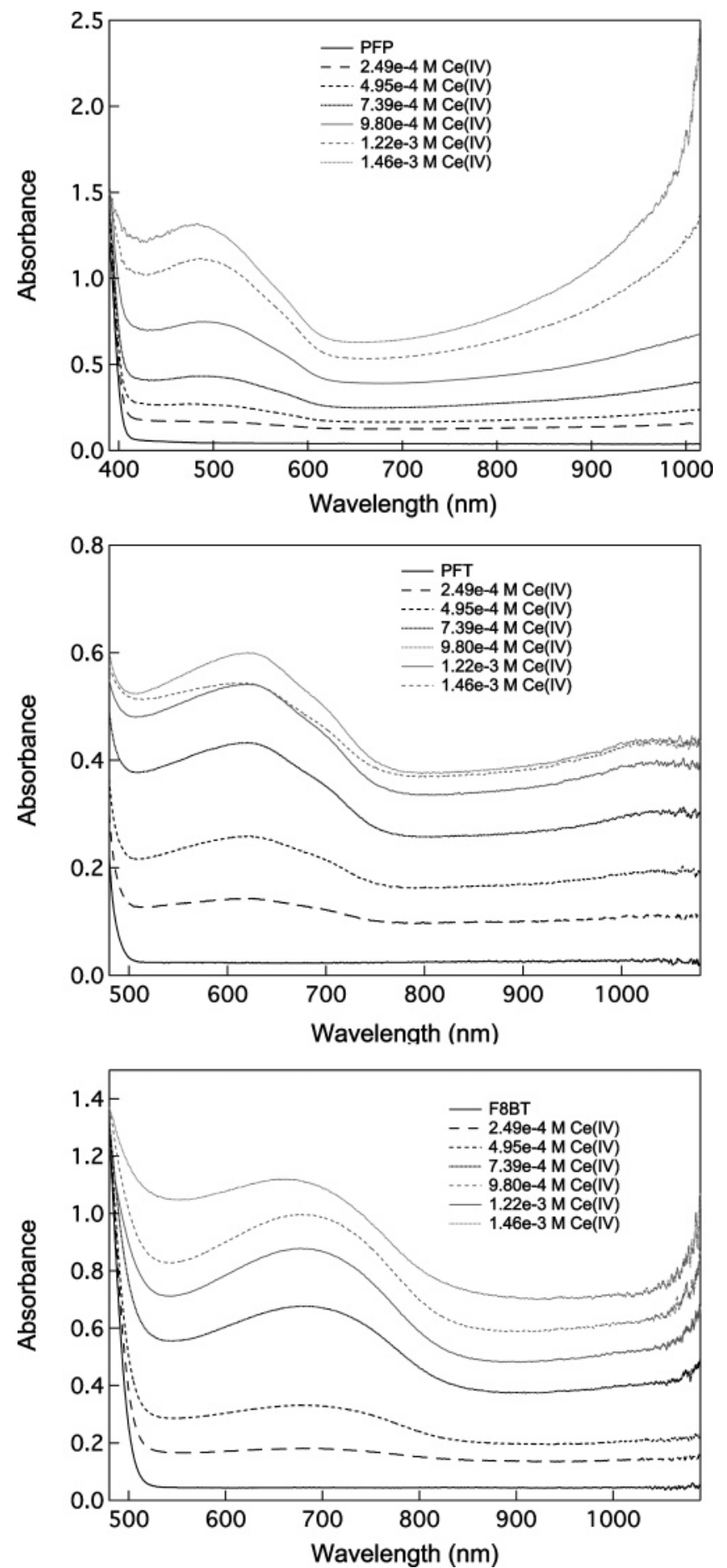

Figure 2. Optical absorption spectra obtained upon oxidation of PFP $\left(8.62 \times 10^{-5} \mathrm{M}\right)$, PFT $\left(5.99 \times 10^{-5} \mathrm{M}\right)$, and F8BT $\left(7.66 \times 10^{-5} \mathrm{M}\right)$ fluorene copolymers in carbon tetrachloride solution by various additions of cerium(IV) ammonium nitrate (in acetonitrile). The data have been offset vertically to facilitate comparison (concentrations in terms of repeat units).

$\mathrm{Ce}(\mathrm{IV})$, these may combine rapidly to form bipolarons. However, the observation of two bands upon oxidation of the copolymers by $\mathrm{Ce}(\mathrm{IV})$ (Figure 2) suggests that singly oxidized species may be formed. However, the fact that these are blueshifted relative to the absorptions seen in the pulse radiolysis experiments may be a consequence of there being more than one polaron on each polymer chain in the chemical oxidation experiments. In support of this, the absorption spectra of dodecathiophenes ${ }^{24}$ with two positive charges on the same chain
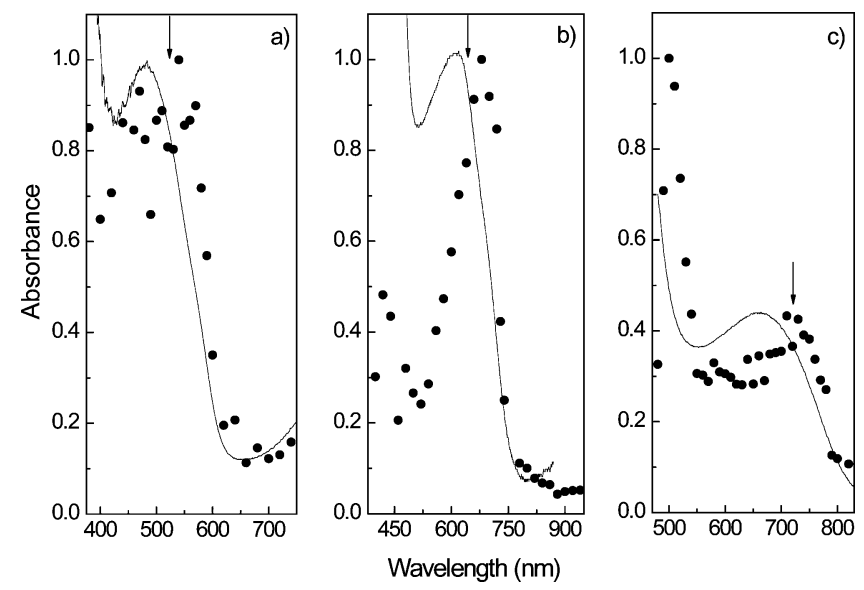

Figure 3. Optical absorption spectra of fluorene copolymer cations in solution obtained by pulse radiolysis (dots) compared with their optical absorption spectra obtained by chemical oxidation (line) (a) PFP, (b) PFT, and (c) F8BT. Values calculated in this paper by TDDFT for the $\mathrm{RC} 2$ band of the trimer are indicated by the arrows.

are significantly blue-shifted relative to the oligomers with a single charge on the same chain. Janssen and co-workers ${ }^{24}$ showed that after the addition of one equivalent of oxidizing species to a dodecathiophene the absorbance of the neutral oligomer decreases only to half the original intensity while that of the oxidized form does not increase as rapidly as in the zone below the 1:1 mole ratio of oxidant to oligomer. The fact that the molar absorption coefficients of the species formed by chemical oxidation in our systems are lower than those formed by pulse radiolysis may have a similar origin and may be associated with a limit to the number of charged species that can be placed on each polymer chain. An alternative explanation for the spectra of the chemically oxidized oligomers is that they may result from degradation products of the initially formed species. In addition, studies in progress show that upon oxidation, fluorene-based systems may undergo chemical degradation via bond cleavage. This may lead to a decrease of the conjugation length, and to the observed blue shift in absorption. Formation of such defects may have implications on the longterm stability of devices containing these polymers.

In contrast, the species produced on pulse radiolysis in carbon tetrachloride can be identified with the initial one-electron positive charge carrier and used for comparison with the theoretical calculations of the spectra.

4.2. Computational Results. To gain more insight in the nature of positive charges on fluorene copolymers and to understand the changes in optical absorption spectra when different moieties are introduced in the fluorene backbone, a DFT study of the charged fluorene co-oligomers has been performed. Geometry deformations, distributions of excess positive and negative charges, and optical absorption spectra were calculated for the cations and anions of the following series of fluorene-based co-oligomers: FPn, FTn, and F8BTn. In the calculations, the substituents at the fluorene 9-position were taken to be hydrogen. This approximation simplifies the calculation without affecting the accuracy. All calculations were performed on symmetric oligomers. The shortest oligomer of each series contains one fluorene unit functionalized with two phenylene, thienylene, and benzothiadiazole units, respectively.

4.2.1. Geometry Deformations and Charge Distributions along Chains of Fluorene-Based Oligomers. The distribution of excess positive and negative charge along chains of fluorenebased oligomers will be discussed in terms of fluorene units (containing $13 \mathrm{C}$-atoms), phenylene units (containing 

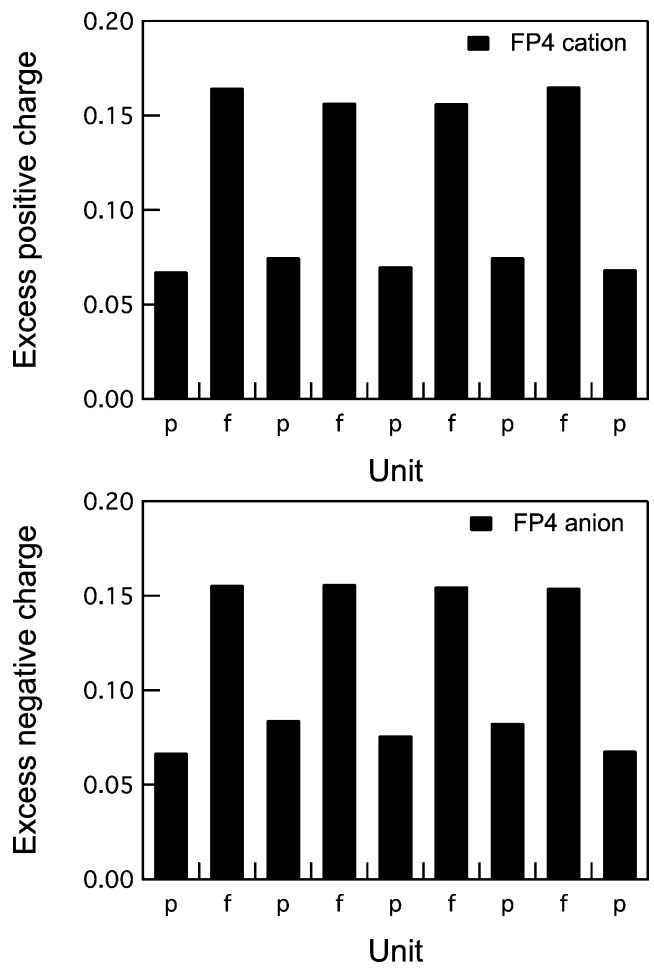

Figure 4. Distribution of an excess positive and negative charge in the FP4 oligomer. Fluorene units are indicated as f, while phenylene units are indicated as $\mathrm{p}$.

$6 \mathrm{C}$-atoms), thienylene units (containing $4 \mathrm{C}$-atoms and one $\mathrm{S}$-atom), and benzothidiazole units (with $6 \mathrm{C}$-atoms, $2 \mathrm{~N}$-atoms and one S-atom). The distribution of an excess positive or negative charge was calculated as the difference between the charges of the atoms in the cations or anions and in the neutral molecules. The DFT results are described in detail below for the three series of oligomers with varying chain length.

FPn Oligomers. The geometries of FPn oligomers were optimized as described in Section 3. Both neutral and charged FPn have a nonplanar structure. For example, the dihedral angle between the fluorene and phenylene units is $42.4^{\circ}$ for neutral FP3. In case of the FP3 cation and anion, the interunit angle becomes smaller: $36.3^{\circ}$ and $31.2^{\circ}$, respectively. In Figure 4, the calculated distribution of an excess positive and negative charge is presented for the FPn tetramer. Similar results were found for the FPn oligomers with other chain lengths. The phenylene units carry a similar amount of charge, which is lower than the charge on the fluorene units. The higher amount of positive charge on the fluorene unit coincides with the lower ionization potential of this unit $\left(7.91 \mathrm{eV}^{49}\right)$ in comparison with that of the phenylene units $\left(9.24 \mathrm{eV}^{50}\right)$. The DFT results suggest that both the excess positive and negative charges are evenly distributed along the chains. Taking into consideration that one fluorene unit consists of two phenylene units, it is found that the positive and negative charges are equally distributed over the FPn chains, indicating a complete delocalization of the charge. This is similar to previous results on phenylenevinylene ${ }^{51,52}$ and fluorene ${ }^{30}$ oligomers.

FTn Oligomers. DFT geometry optimization leads to a smaller value for the inter-unit angle of FTn oligomers than for FPn oligomers. For FT3 neutral, cation, and anion, the angles are $27.9^{\circ}, 23.7^{\circ}$, and $20.3^{\circ}$, respectively. As found for FPn oligomers, the interunit angle decreases slightly upon introduction of a charge. The distribution of an excess charge on a FT4 oligomer (Figure 5) is similar to that for FP4 (see Figure 4). Interestingly, the distribution of a positive charge along the FT4
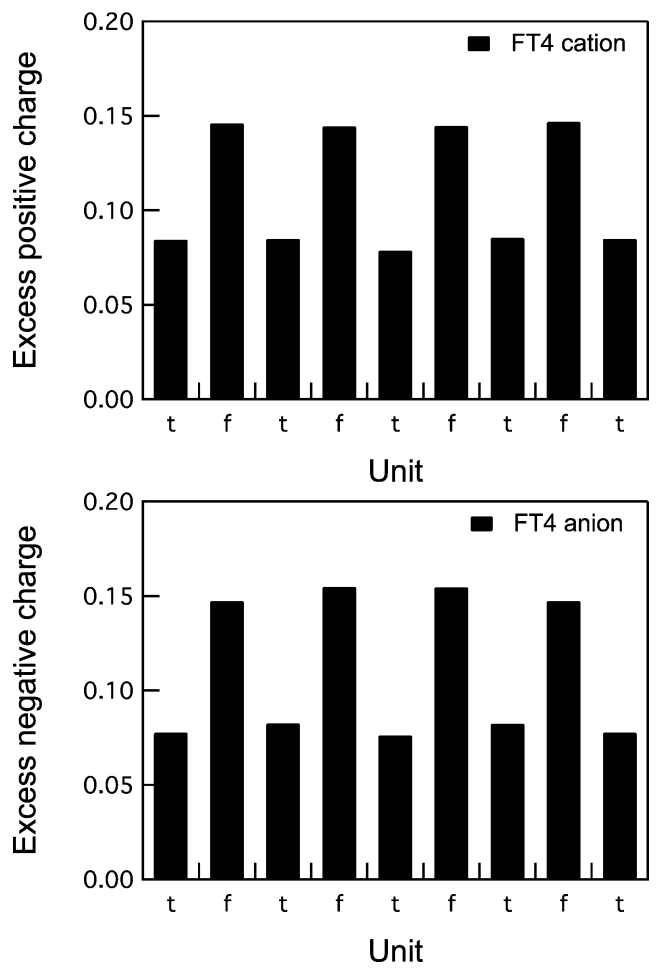

Figure 5. Distribution of an excess positive and negative charge in the FT4 oligomer. Fluorene units are indicated as f, while thienylene units are indicated as $\mathrm{t}$.

chain is similar to that of the negative charge. The charge is delocalized along the entire chain, with the fluorene units carrying a higher amount of charge than the thienylene units. When considering the positive charge per ring for the fluorene and thienylene units, it is found that the charge is delocalized over these rings, as was also found for FP4. It is seen in Figure 5 that the amount of charge on a fluorene unit is about twice that on a thiophene unit. Hence, the amount of charge per ring is similar, which indicates an even distribution of the charge. This effect is also found for FTn anions. The even distribution of the charge over the co-oligomer chains reflects the fact that the charge does not induce a local geometrical deformation; polaron formation does not occur according to the DFT calculations.

F8BTn Oligomers. The DFT geometry optimization shows that the interunit angle for the F8BT1 oligomer in its neutral state is $45.5^{\circ}$, the cation has an interunit angle of $35.9^{\circ}$, while the angle for the F8BT1 anion is $41.9^{\circ}$. This is in contrast with the FPn and FTn oligomers in which the interunit angle corresponding to the cation is larger than the angle for the anion. When the thienylene or phenylene units are replaced with benzothiadiazole units, the charge distribution of an excess positive charge is quite different from the charge distribution of an excess negative charge. This is because of the strong electron-accepting character of the benzothiadiazole unit. The DFT charge distribution of the positively charged F8BTn tetramer shows that the fluorene units carry a higher amount of charge than the benzothiadiazole units (see the first graph of Figure 6 ). About $58 \%$ of the positive charge is distributed over the fluorene units, while the benzothiadiazole units carry $42 \%$. In contrast, for the F8BT4 anion the highest amount of charge is present on the benzothiadiazole units $(70 \%)$ (see the second graph of Figure 6). Moreover, the excess negative charge is not uniformly distributed over the benzothiadiazole units, but the charge has a tendency to localize toward the edges of the tetramer. 

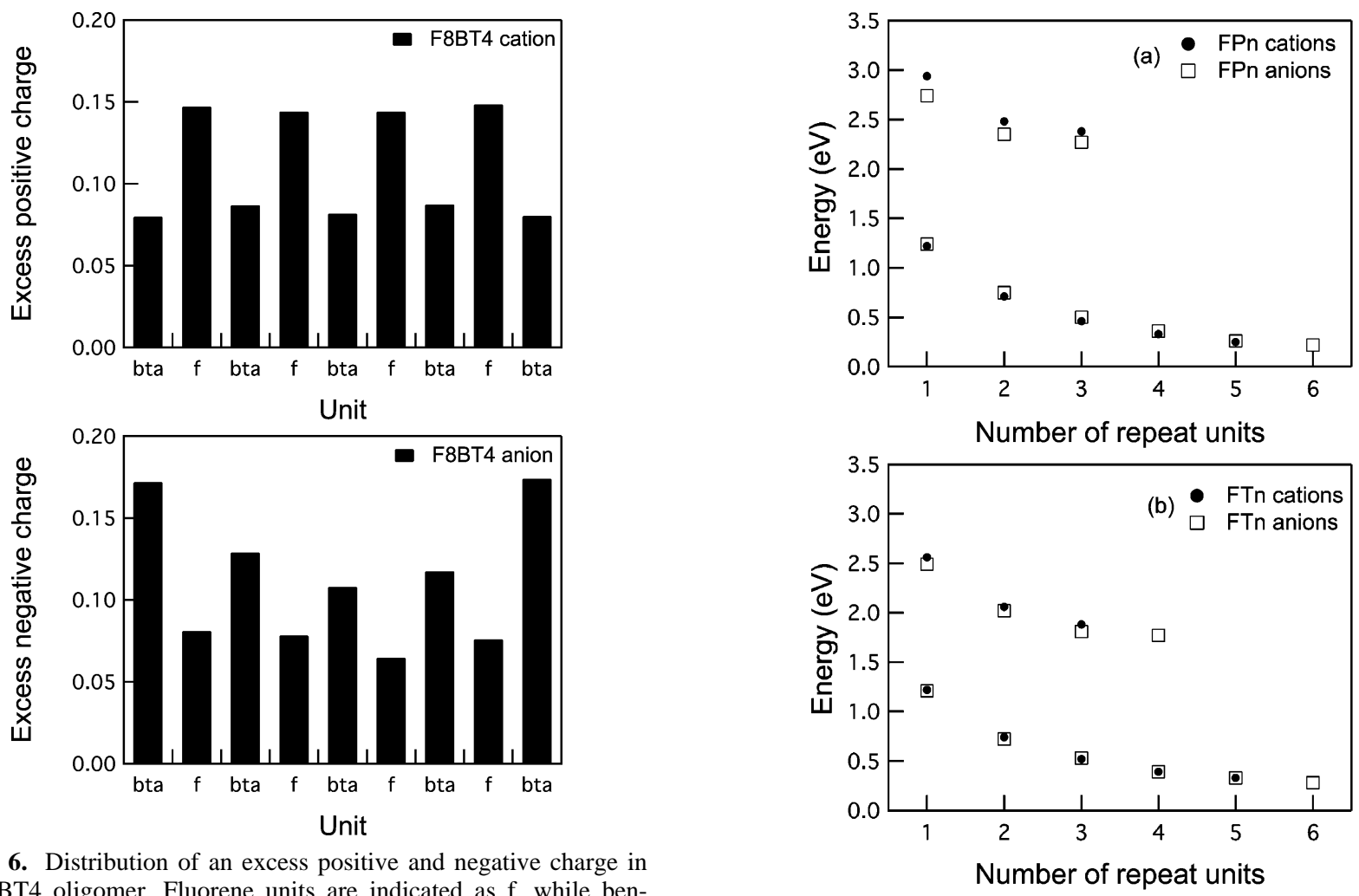

Figure 6. Distribution of an excess positive and negative charge in the F8BT4 oligomer. Fluorene units are indicated as f, while benzothiadiazole units are indicated as bta.

TABLE 2: Calculated Transition Energies, Oscillator Strengths, and Relative Contribution from Excited-State Configuration for Cations of FPn Oligomers (Only the Transitions with an Oscillator Strength Higher than 0.1 Are Given)

\begin{tabular}{ccccc}
\hline oligomer & band & $\begin{array}{c}\text { energy } \\
(\mathrm{eV})\end{array}$ & $\begin{array}{c}\text { oscillator } \\
\text { strength }\end{array}$ & $\begin{array}{c}\text { relative contribution } \\
\text { of excited-state configuration }\end{array}$ \\
\hline FP1 & RC1 & 1.22 & 0.42 & $0.98(\mathrm{H} \rightarrow \mathrm{P} 1)$ \\
& RC2 & 2.94 & 0.71 & $0.91(\mathrm{P} 1 \rightarrow \mathrm{P} 2)$ \\
FP2 & RC1 & 0.71 & 0.85 & $1.13(\mathrm{H} \rightarrow \mathrm{P} 1)$ \\
& RC2 & 2.48 & 0.79 & $0.92(\mathrm{P} 1 \rightarrow \mathrm{P} 2)$ \\
FP3 & RC1 & 0.46 & 0.99 & $1.25(\mathrm{H} \rightarrow \mathrm{P} 1)$ \\
& RC2 & 2.38 & 0.67 & $0.94(\mathrm{P} 1 \rightarrow \mathrm{P} 2)$ \\
FP4 & RC1 & 0.33 & 1.04 & $1.37(\mathrm{H} \rightarrow \mathrm{P} 1)$ \\
FP5 & RC1 & 0.25 & 0.98 & $1.51(\mathrm{H} \rightarrow \mathrm{P} 1)$
\end{tabular}

4.2.2. Calculated Optical Absorption Spectra of Charged Fluorene-Based Oligomers. Optical absorption spectra have been calculated for the three series of oligomers using TDDFT.

FPn Oligomers. The calculated transition energies for FPn cations are presented in Table 2 together with the oscillator strength and the composition of the excited states. In Figure $7 \mathrm{a}$, the absorption maxima of FPn cations are shown as a function of chain length. The maximum of absorption decreases with increasing chain length, indicating delocalization of the positive charge along the chain. A similar monotonous decrease of the transition energies with increasing chain length was previously found from TDDFT calculations on phenylenevinylene ${ }^{51,52}$ and fluorene ${ }^{30}$ cations.

The energy of the RC1 transition decreases from $1.22 \mathrm{eV}$ for the FP1 cation to a value of $0.46 \mathrm{eV}$ for the FP3 cation, while the RC2 transition energy shifts from 2.94 to $2.38 \mathrm{eV}$. This indicates that the low-energy $\mathrm{RC} 1$ band is more sensitive to chain length than the RC2 band. According to the TDDFT calculations the RC1 transition is most intense (has the highest oscillator strength) except for the shortest oligomer (FP1 cation). The optical absorption spectra of FPn cations can be discussed using a molecular orbital model, shown schematically in Figure

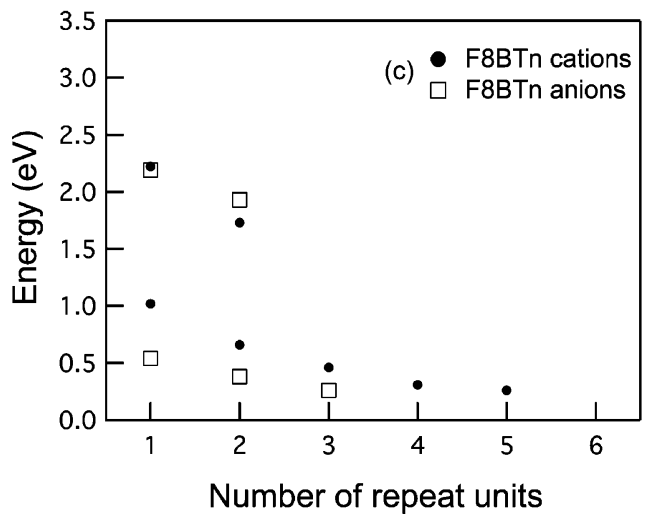

Figure 7. Chain-length dependence of the transition energies for cations and anions of FPn, FTn, and F8BTn oligomers.

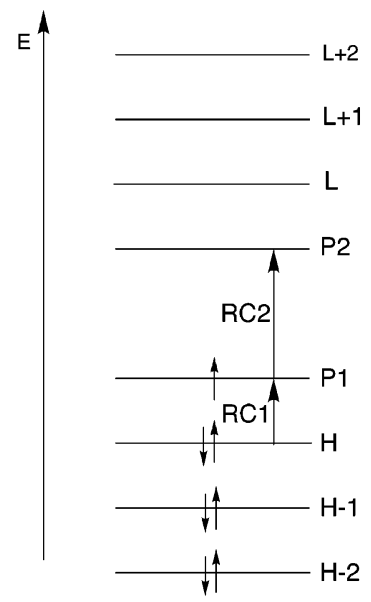

(a)

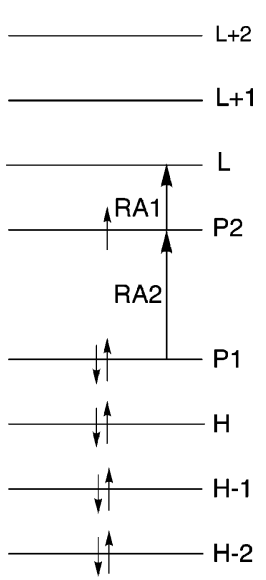

(b)

Figure 8. Molecular orbital model for (a) cations and (b) anions for fluorene-based oligomers. The electronic transitions are also represented.

8a. This model considers that removal of an electron introduces additional transitions with lower energy than the one of the 
TABLE 3: Calculated Transition Energies, Oscillator Strengths, and Relative Contribution from Excited-State Configuration for Anions of FPn Oligomers (Only the Transitions with an Oscillator Strength Higher than 0.1 Are Given)

\begin{tabular}{ccccc}
\hline oligomer & band & $\begin{array}{c}\text { energy } \\
(\mathrm{eV})\end{array}$ & $\begin{array}{c}\text { oscillator } \\
\text { strength }\end{array}$ & $\begin{array}{c}\text { relative contribution } \\
\text { of excited-state configuration }\end{array}$ \\
\hline FP1 & RA1 & 1.24 & 0.46 & $0.98(\mathrm{P} 2 \rightarrow \mathrm{L})$ \\
& RA2 & 2.74 & 0.80 & $-0.89(\mathrm{P} 1 \rightarrow \mathrm{P} 2)$ \\
FP2 & RA1 & 0.75 & 0.96 & $1.09(\mathrm{P} 2 \rightarrow \mathrm{L})$ \\
& RA2 & 2.35 & 0.99 & $0.97(\mathrm{P} 1 \rightarrow \mathrm{P} 2)$ \\
FP3 & RA1 & 0.50 & 1.16 & $-1.20(\mathrm{P} 2 \rightarrow \mathrm{L})$ \\
& RA2 & 2.27 & 0.81 & $0.96(\mathrm{P} 1 \rightarrow \mathrm{P} 2)$ \\
FP4 & RA1 & 0.36 & 1.21 & $1.32(\mathrm{P} 2 \rightarrow \mathrm{L})$ \\
FP5 & RA1 & 0.26 & 1.13 & $1.45(\mathrm{P} 2 \rightarrow \mathrm{L})$ \\
FP6 & RA1 & 0.22 & 1.28 & $1.51(\mathrm{P} 2 \rightarrow \mathrm{L})$
\end{tabular}

neutral compound. Because the molecular orbital model is derived from the polaron model, ${ }^{53}$ the energy levels between additional transitions will be named after the polaronic levels, P1 and P2. According to this model, in FPn cations the P1 level is singly occupied, while P2 represents the lowest unoccupied molecular orbital. The highest doubly occupied molecular orbital is indicated as $\mathrm{H}$, while the second empty level for cations is denoted as L (see Figure 8a). In the last column of Table 2, the relative contributions of excited-state configurations are presented in terms of this model. The lowest transition (RC1) of FPn cations is dominated by the configuration in which an electron from the highest doubly occupied molecular orbital is excited to the singly occupied molecular orbital $(\mathrm{H} \rightarrow \mathrm{P} 1)$. Experimentally, it was not possible to see the lowest energy transition of the copolymer cations in the pulse radiolysis measurements due to lack of suitable detectors for the near-IR region. However, evidence of its presence comes from the chemical oxidation studies. The second RC2 transition corresponds mainly to excitation of an electron from the singly occupied molecular orbital to the LUMO $(\mathrm{P} 1 \rightarrow \mathrm{P} 2)$.

The optical transition energies obtained from calculations on FPn anions are close to those for the cations, although the nature of the transitions is different. The maxima of the optical absorption shift as a function of chain lengths as shown in Table 3 and Figure 7a. The energy of the lowest transition (RA1) decreases from $1.24 \mathrm{eV}$ for the FP1 anion to $0.50 \mathrm{eV}$ for the FP3 anion. This shift of the RA1 transition energy with chain length is larger than for the RA2 transition (see Table 3). The red shift of the RA1 transition continues up to the longest cooligomer considered. Hence, according to the TDDFT calculations the energy of the RA1 transition is a sensitive probe to the delocalization of the charge. The optical absorption transitions of phenylene fluorene anions are explained using the molecular orbital model illustrated in Figure 8b. According to the TDDFT results, the low-energy band (RA1) in the optical absorption spectra of FPn anions is due to an excitation of an electron from the singly occupied molecular orbital (P2) to the LUMO (L): $(\mathrm{P} 2 \rightarrow \mathrm{L})$. The second transition in the spectra from Table 3 corresponds mainly to a transition of an electron from the highest doubly occupied molecular orbital (P1) to the singly occupied molecular orbital $(\mathrm{P} 2):(\mathrm{P} 1 \rightarrow \mathrm{P} 2)$. The composition of excited states of FPn anions and cations is very similar to those calculated for charged phenylenevinylene ${ }^{52,54}$ and fluorene oligomers. ${ }^{30}$

The RC2 and RA2 transitions could not be calculated for FPn $(n>3)$ because of the limited computer memory available. The similarity between the results obtained for FPn cations and anions is reflected both in the optical absorption spectra and in the charge distribution.
TABLE 4: Calculated Transition Energies, Oscillator Strengths, and Relative Contribution from Excited-State Configuration for Cations of FTn Oligomers (Only the Transitions with an Oscillator Strength Higher than 0.1 Are Given)

\begin{tabular}{ccccc}
\hline oligomer & band & $\begin{array}{c}\text { energy } \\
(\mathrm{eV})\end{array}$ & $\begin{array}{c}\text { oscillator } \\
\text { strength }\end{array}$ & $\begin{array}{c}\text { relative contribution } \\
\text { of excited-state configuration }\end{array}$ \\
\hline FT1 & RC1 & 1.22 & 0.37 & $0.99(\mathrm{H} \rightarrow \mathrm{P} 1)$ \\
& RC2 & 2.56 & 0.91 & $0.94(\mathrm{P} 1 \rightarrow \mathrm{P} 2)$ \\
FT2 & RC1 & 0.74 & 0.83 & $0.62(\mathrm{H} \rightarrow \mathrm{P} 1)$ \\
& RC2 & 2.06 & 0.85 & $0.92(\mathrm{P} 1 \rightarrow \mathrm{P} 2)$ \\
FT3 & RC1 & 0.52 & 1.16 & $1.18(\mathrm{H} \rightarrow \mathrm{P} 1)$ \\
& RC2 & 1.88 & 0.85 & $0.90(\mathrm{P} 1 \rightarrow \mathrm{P} 2)$ \\
FT4 & RC1 & 0.39 & 1.33 & $1.28(\mathrm{H} \rightarrow \mathrm{P} 1)$ \\
FT5 & RC1 & 0.33 & 1.73 & $-1.35(\mathrm{H} \rightarrow \mathrm{P} 1)$
\end{tabular}

TABLE 5: Calculated Transition Energies, Oscillator Strengths, and Relative Contribution from Excited-State Configuration for Anions of FTn Oligomers (Only the Transitions with an Oscillator Strength Higher than 0.1 Are Given)

\begin{tabular}{ccccc}
\hline oligomer & band & $\begin{array}{c}\text { energy } \\
(\mathrm{eV})\end{array}$ & $\begin{array}{c}\text { oscillator } \\
\text { strength }\end{array}$ & $\begin{array}{c}\text { relative contribution } \\
\text { of excited-state configuration }\end{array}$ \\
\hline FT1 & RA1 & 1.21 & 0.35 & $0.98(\mathrm{P} 2 \rightarrow \mathrm{L})$ \\
& RA2 & 2.49 & 0.63 & $0.79(\mathrm{P} 1 \rightarrow \mathrm{P} 2)+0.54(\mathrm{P} 2 \rightarrow \mathrm{L}+6)$ \\
$\mathrm{FT} 2$ & RA1 & 0.72 & 0.78 & $1.08(\mathrm{P} 2 \rightarrow \mathrm{L})$ \\
& RA2 & 2.02 & 1.07 & $0.96(\mathrm{P} 1 \rightarrow \mathrm{P} 2)$ \\
$\mathrm{FT} 3$ & RA1 & 0.53 & 1.17 & $-1.15(\mathrm{P} 2 \rightarrow \mathrm{L})$ \\
& RA2 & 1.81 & 1.01 & $0.94(\mathrm{P} 1 \rightarrow \mathrm{P} 2)$ \\
FT4 & RA1 & 0.39 & 1.34 & $1.25(\mathrm{P} 2 \rightarrow \mathrm{L})$ \\
& RA2 & 1.77 & 0.91 & $0.95(\mathrm{P} 1 \rightarrow \mathrm{P} 2)$ \\
FT5 & RA1 & 0.33 & 1.66 & $-1.32(\mathrm{P} 2 \rightarrow \mathrm{L})$ \\
FT6 & RA1 & 0.28 & 1.83 & $1.39(\mathrm{P} 2 \rightarrow \mathrm{L})$
\end{tabular}

TABLE 6: Calculated Transition Energies, Oscillator Strengths, and Relative Contribution from Excited-State Configuration for Cations of F8BTn Oligomers (Only the Transitions with an Oscillator Strength Higher than 0.1 Are Given)

\begin{tabular}{ccccc}
\hline oligomer & band & $\begin{array}{c}\text { energy } \\
(\mathrm{eV})\end{array}$ & $\begin{array}{c}\text { oscillator } \\
\text { strength }\end{array}$ & $\begin{array}{c}\text { relative contribution } \\
\text { of excited-state configuration }\end{array}$ \\
\hline F8BT1 & RC1 & 1.02 & 0.41 & $1.02(\mathrm{H} \rightarrow \mathrm{P} 1)$ \\
& RC2 & 2.22 & 0.24 & $0.85(\mathrm{P} 1 \rightarrow \mathrm{P} 2)$ \\
F8BT2 & RC1 & 0.66 & 0.81 & $1.10(\mathrm{H} \rightarrow \mathrm{P} 1)$ \\
& RC2 & 1.73 & 0.41 & $0.89(\mathrm{P} 1 \rightarrow \mathrm{P} 2)$ \\
F8BT3 & RC1 & 0.46 & 1.02 & $1.20(\mathrm{H} \rightarrow \mathrm{P} 1)$ \\
F8BT4 & RC1 & 0.31 & 0.91 & $1.37(\mathrm{H} \rightarrow \mathrm{P} 1)$ \\
F8BT5 & RC1 & 0.26 & 1.11 & $1.45(\mathrm{H} \rightarrow \mathrm{P} 1)$
\end{tabular}

FTn Oligomers. The results obtained for FTn oligomers are similar to those obtained for FPn oligomers. Both cations and anions of the FTn oligomers exhibit two absorption bands, whose energies decrease with increasing chain length. The values of the absorption maxima are shown in Tables 4 and 5 and in Figure 7b. The RC1 and RC2 transition energies shift by a close to equal amount on going from the FT1 to FT3, despite the different nature of these transitions. The RC1 transition is mainly due to an $\mathrm{H} \rightarrow \mathrm{P} 1$ excitation, while $\mathrm{RC} 2$ is dominated by a $\mathrm{P} 1 \rightarrow \mathrm{P} 2$ excitation. The absorption features for FTn anions are very similar to those for the corresponding cations. However, the nature of the optical transitions for the anions is different from that for cations. For anions, the lowenergy transition (RA1) is dominated by a P $2 \rightarrow \mathrm{L}$ excitation, while the high-energy transition (RA2) corresponds to a P1 $\rightarrow$ P2 excitation.

F8BTn Oligomers. The absorption maxima of F8BTn cations and anions are presented in Tables 6 and 7, respectively, and in Figure 7c. For the F8BT1 cation, the low-energy band (RC1) is at $1.02 \mathrm{eV}$, while the F8BT1 anion absorbs at $0.54 \mathrm{eV}$ (RA1). The energy difference of approximately $0.5 \mathrm{eV}$ between RA1 
TABLE 7: Calculated Transition Energies, Oscillator Strengths, and Relative Contribution from Excited-State Configuration for Anions of F8BTn Oligomers (Only the Transitions with an Oscillator Strength Higher than 0.1 Are Given)

\begin{tabular}{ccccl}
\hline oligomer & band & $\begin{array}{c}\text { energy } \\
(\mathrm{eV})\end{array}$ & $\begin{array}{c}\text { oscillator } \\
\text { strength }\end{array}$ & \multicolumn{1}{c}{$\begin{array}{c}\text { relative contribution } \\
\text { of excited-state configuration }\end{array}$} \\
\hline F8BT1 & RA1 & 0.54 & 0.18 & $1.35(\mathrm{P} 2 \rightarrow \mathrm{L})$ \\
& RA2 & 2.19 & 0.12 & $0.83(\mathrm{P} 1 \rightarrow \mathrm{P} 2)$ \\
F8BT2 & RA1 & 0.38 & 0.34 & $1.43(\mathrm{P} 2 \rightarrow \mathrm{L})$ \\
& RA2 & 1.93 & 0.14 & $0.64(\mathrm{P} 1 \rightarrow \mathrm{P} 2)+0.56(\mathrm{P} 1 \rightarrow \mathrm{L}+1)$ \\
F8BT3 & RA1 & 0.26 & 0.40 & $1.57(\mathrm{P} 2 \rightarrow \mathrm{L})$
\end{tabular}

and $\mathrm{RC} 1$ as found for F8BT1 was not observed for the other fluorene series. This energy difference between the F8BT1 cation and anion absorption can be correlated with the very different charge distribution for the anion and cation, as discussed in Section 4.2. It was shown that for F8BTn cations the charge is localized on the fluorene units, while for F8BTn anions most of the charge is distributed over the benzothiadiazole units. This is reflected in different values of the absorption energy of the F8BTn cations and anions. This is in contrast with the results found for FPn and FTn oligomers in which the positive and negative charges are localized on the same unit (fluorene), leading to a similar cation and anion spectra. The energy difference between the transitions for F8BTn cations and anions decreases with increasing chain length. This is explained by the fact that the amount of negative charge localized on benzothiadiazole units decreases with increasing chain length from $80 \%$ for the F8BT1 anion to $68 \%$ for the F8BT5 anion, while the amount of positive charge on the benzothiadiazole units is close to constant (58\%) for all chain lengths.

The optical absorption spectra of charged fluorenes have been calculated previously using TDDFT. ${ }^{30}$ The comparison of fluorene spectra with the present calculations leads to the conclusion that the absorption maxima for the fluorene cooligomers are red-shifted.

In Figure 9, the high-energy band (RC2) of cations of FPn, FTn, and F8BTn co-oligomers is plotted as a function of the reciprocal value of the number of repeat units. Linear extrapolation of the calculated data on co-oligomers gives absorption energies for the radical cations of PFP, PFT, and F8BT copolymers at $2.08 \mathrm{eV}(600 \mathrm{~nm}), 1.55 \mathrm{eV}(800 \mathrm{~nm})$, and 1.24 $\mathrm{eV}(1000 \mathrm{~nm})$, respectively. As discussed in Section 4.1 and presented in Table 1, the energies measured in the chemical oxidation experiments for the PFP, PFT, and F8BT copolymers are $2.58 \mathrm{eV}(480 \mathrm{~nm}), 2.00 \mathrm{eV}(620 \mathrm{~nm})$, and $1.82 \mathrm{eV}(680 \mathrm{~nm})$. The results of the pulse radiolysis experiments are $2.30 \mathrm{eV}(540$ $\mathrm{nm})$ for the PFP radical cation, $1.82 \mathrm{eV}(680 \mathrm{~nm})$ for the PFT radical cation, and $1.72 \mathrm{eV}(720 \mathrm{~nm})$ for the $\mathrm{F} 8 \mathrm{BT}$ radical cation. Comparison of the extrapolated values with those determined by chemical oxidation and pulse radiolysis shows that the calculated transition energies are closest to those found in the pulse radiolysis experiments (only the F8BT cation has the absorption maximum approximately $280 \mathrm{~nm}$ lower than found in the pulse radiolysis experiments).

The linear extrapolation of the calculated data yields transition energies that are lower than the experimental data for the copolymers. The difference between the extrapolated results and the pulse radiolysis data reflects the approximate nature of the assumed linear dependence of the absorption energy on the reciprocal of the number of repeat units. Considerable deviations from such a linear dependence can be expected if the charge is not fully delocalized along the chain. Such (partial) localization of the charge can occur because of structural disorder. For the copolymers studied here, there is expected to be substantial

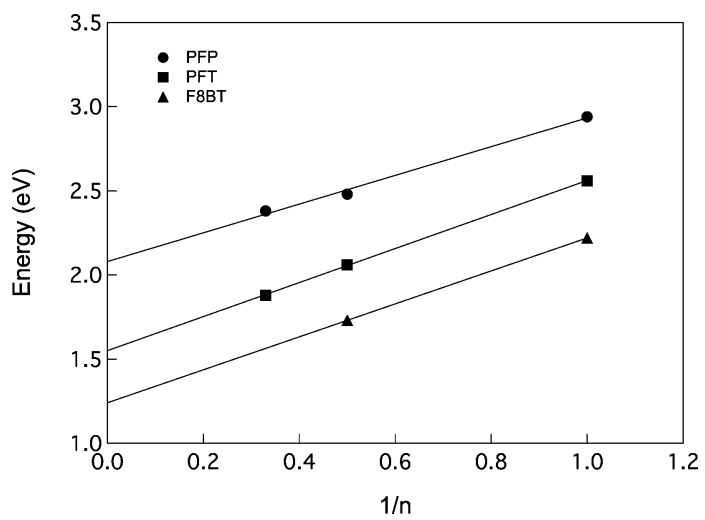

Figure 9. RC2 transition energies plotted against the inverse chain length for FPn, FTn, and F8BTn. The extrapolation of the linear fit gives the RC2 transition energy for an infinite chain (the corresponding copolymer).

disorder in the rotation angles between neighboring units because the rotation potential is expected to be relatively low. ${ }^{30}$ It is interesting that the calculated values for the RC2 band of the trimers are very close to those observed by pulse radiolysis, as indicated by the arrows in Figure 3, supporting a model of relatively localized charged species. An additional source of localization leading to deviation from a linear behavior can be the self-localization of the charge leading to a polaron.

\section{Conclusions}

In this paper, we describe a combined experimental and theoretical study of structural and opto-electronic properties of charged fluorene-based oligomers and polymers. Radical cations of fluorene copolymers were produced by chemical oxidation with $\mathrm{Ce}(\mathrm{IV})$ and by pulse radiolysis. Differences in both spectral and kinetic behavior were observed. It is believed that the species observed in the chemical oxidation experiments correspond to products formed following decay of the initial oneelectron oxidized charged species. However, the introduction of different moieties to the fluorene backbone was found to have a large effect on the optical absorption spectra of the copolymer cations.

DFT calculations on the geometry optimization of charged oligomers show nonplanar structures. An analysis of the charge distribution along the fluorene-based oligomer chains show that for FPn and FTn the charge is evenly distributed over the fluorene units. Similar behavior has been found for F8BTn cations. In contrast, the excess negative charge of F8BTn oligomers is localized on the benzothiadiazole units with higher amounts on the edges of the chain. This is explained by the electron-accepting character of the benzothiadiazole moieties.

TDDFT calculations of the cation and anion optical absorption spectra show a monotonous decrease of the low-energy band with increasing chain length. This indicates the delocalization of charge along the chains. RA1 absorption band of F8BTn anions has a lower energy than the RC1 band of F8BTn cations. This correlates with the different distribution of the charge in F8BTn anions and cations. When the positive and negative charges are localized on different units (as in F8BTn cations and anions) the optical absorption spectra are different. If charges are localized on the same units (fluorene units in the case of FPn and FTn cations and anions) similar absorption spectra are obtained.

The absorption maxima calculated for cations of the fluorene co-oligomers are red-shifted compared with those for oligomers containing fluorene units only. 
Data of the absorption maxima of the positively charged fluorene-based oligomers have been extended to data on polymers. The extrapolation of high-energy data of the oligomers indicates the following absorption maxima: $2.08 \mathrm{eV}(600 \mathrm{~nm})$, $1.55 \mathrm{eV}(800 \mathrm{~nm})$, and $1.24 \mathrm{eV}(1000 \mathrm{~nm})$ for PFP, PFT, and F8BT, respectively, which are in reasonable agreement with the values from pulse radiolysis experiments. A better agreement is observed with calculations for the trimers, suggesting that the charges are not completely delocalized.

TDDFT calculations give also indications about the energy levels in between which the electronic transitions take place. In this context, TDDFT predicts that the RC1 transition is dominated by an excitation of an electron from $\mathrm{H} \rightarrow \mathrm{P} 1, \mathrm{RC} 2$ corresponds to a $\mathrm{P} 1 \rightarrow \mathrm{P} 2$ excitation, RA1 to $\mathrm{P} 2 \rightarrow \mathrm{L}$, and RA2 to $\mathrm{P} 1 \rightarrow \mathrm{P} 2$, in agreement with previous results found for different-charged oligomers.

Acknowledgment. Pulse radiolysis experiments were carried out at the Free Radical Research Facility in the Synchrotron Radiation Department of the CLRC Daresbury Laboratory, Warrington, UK, with the support of the European Commission through the "Improving Human Potential" Transnational Access to Major Research Infrastructures Contract HPRI-CT-200200183. We are indebted to Drs. S. Navaratnam and R. Edge for their excellent technical support and Dr. J. Seixas de Melo, A. A. Kharlamov, and Mr. J. Pina for further assistance. We thank the Fundação para a Ciência e a Tecnologia (FCT) for the award of a postdoctoral fellowship to S.M.F. (grant SFRH/ BPD/14924/2004). The Portuguese groups thank POCI, FCT, and FEDER for further financial support. F.C.G. acknowledges the "Nederlandse Organisatie voor Wetenschappelijk Onderzoek (NWO)" for financial support in the form of a VENI grant.

Supporting Information Available: Decay kinetics of the cation of PFT copolymer. This material is available free of charge via the Internet at http://pubs.acs.org.

\section{References and Notes}

(1) Nijegorodov, N. I.; Downey, W. S. J. Phys. Chem. 1994, 98, 5639. (2) Yang, L.; Liao, Y.; Feng, J.-K.; Ren, A.-M. J. Phys. Chem. A 2005 109, 7764

(3) Belletete, M.; Beaupre, S.; Bouchard, J.; Blondin, P.; Leclerc, M.; Durocher, G. J. Phys. Chem. B 2000, 104, 9118.

(4) Belletete, M.; Morin, J.-F.; Beaupre, S.; Leclerc, M.; Durocher, G. Synth. Met. 2002, 126, 43.

(5) Tirapattur, S.; Belletete, M.; Leclerc, M.; Durocher, G. J. Mol. Struct. (THEOCHEM) 2003, 625, 141.

(6) Gong, Z.; Lagowski, J. B. J. Mol. Struct. (THEOCHEM) 2005 , 729, 211.

(7) Belletete, M.; Morin, J.-F.; Beaupre, S.; Ranger, M.; Leclerc, M.; Durocher, G. Macromolecules 2001, 34, 2288

(8) Poolmee, P.; Ehara, M.; Hannongbua, S.; Nakatsuji, H. Polymer 2005, 46, 6474 .

(9) Yang, L.; Feng, J.-K.; Ren, A.-M. J. Mol. Struct. (THEOCHEM) 2006, 758,29

(10) Grell, M.; Redecker, M.; Whitehead, K. S.; Bradley, D. D. C.; Inbasekaran, M.; Woo, E. P.; Wu, W. Liq. Cryst. 1999, 26, 1403.

(11) Donley, C. L.; Zaumseil, J.; Andreasen, J. W.; Nielsen, M. M.; Sirringhaus, H.; Friend, R. H.; Kim, J.-S. J. Am. Chem. Soc. 2005, 127, 12890 .

(12) Cornil, J.; Gueli, I.; Dkhissi, A.; Sancho-Garcia, J. C.; Hennebicq, E.; Calbert, J. P.; Lemaur, V.; Beljonne, D.; Bredas, J. L. J. Chem. Phys. 2003, 118, 6615 .

(13) Donat-Bouillud, A.; Levesque, I.; Tao, Y.; D'Iorio, M.; Beaupre, S.; Blondin, P.; Ranger, M.; Bouchard, J.; Leclerc, M. Chem. Mater. 2000, $12,1931$.

(14) Beaupre, S.; Leclerc, M. Adv. Funct. Mater. 2002, 12, 192.

(15) Kim, Y.; Bradley, D. D. C. Curr. Appl. Phys. 2005, 5, 222

(16) Sprangler, C. W.; Hall, T. J.; Sapochak, L. S.; Liu, P.-K. Polymer 1989, $30,1166$.
(17) Deussen, M.; Bassler, H. Chem. Phys. 1992, 164, 247.

(18) Sakamoto, A.; Furukawa, Y.; Tasumi, M. J. Phys. Chem. 1994, $98,4635$.

(19) Schenk, R.; Gregorius, H.; Mullen, K. Adv. Mater. 1991, 3, 492.

(20) Furukawa, Y.; Sakamoto, A.; Tasumi, M. Macromol. Symp. 1996, 101,95 .

(21) Oberski, J. M.; Greiner, A.; Bassler, H. Chem. Phys. Lett. 1991, $184,391$.

(22) Schenk, R.; Gregorius, H.; Meerholz, K.; Heinze, J.; Mullen, K. J. Am. Chem. Soc. 1991, 113, 2634.

(23) Gebhardt, V.; Bacher, A.; Thelakkat, M.; Stalmach, U.; Meier, H.; Schmidt, H.-W.; Haarer, D. Adv. Mater. 1999, 11, 119.

(24) van Haare, J. A. E. H.; Havinga, E. E.; van Dongen, J. L. J.; Janssen,

R. A. J.; Cornil, J.; Bredas, J.-L. Chem.-Eur. J. 1998, 4, 1509.

(25) Scott, J. C.; Kaufman, J. H.; Brock, P. J.; DiPietro, R.; Salem, J.; Goita, J. A. J. Appl. Phys. 1996, 79, 2745.

(26) Gamertih, S.; Nothover, H.-G.; Scherf, U.; List, E. J. W. Jpn. J. Appl. Phys. 2004, 43, L891.

(27) Narwark, O.; Peetz, R.; Thorn-Csanyi, E.; Burrows, H. D.; Monkman, A. P.; Hamblett, I.; Navaratnam, S. Chem. Mater., submitted for publication, 2006

(28) Hoofman, R. O. M.; de Haas, M. P.; Siebbeles, L. D. A.; Warman, J. M. Nature 1998, 392, 54.

(29) Burrows, H. D.; da Miguel, G. M.; Monkman, A. P.; Horsburgh, L. E.; Hamblett, I.; Navaratnam, S. J. Chem. Phys. 2000, 112, 3082.

(30) Fratiloiu, S.; Grozema, F. C.; Koizumi, Y.; Seki, S.; Saeki, A.; Tagawa, S.; Dudek, S. P.; Siebbeles, L. D. A. J. Phys. Chem. B 2006, 110 5984.

(31) Asawapirom, U.; Guntner, R.; Forster, M.; Farrell, T.; Scherf, U. Synthesis 2002, 9, 1136.

(32) Charas, A.; Morgado, J.; Martinho, J. M. G.; Alcacer, L.; Lim, S. F.; Friend, R. H.; Cacialli, F. Polymer 2003, 44, 1843.

(33) Fonseca, S. M.; Pina, J.; Arnaut, L. G.; Seixas de Melo, J.; Burrows, H. D.; Chattopadhyay, N.; Alcacer, L.; Charas, A.; Morgado, J.; Monkman, A. P.; Asawapirom, U.; Scherf, U.; Edge, R.; Navaratnam, S. J. Phys. Chem. $B$ 2006, 110, 8278.

(34) Burrows, H. D.; Greatorex, D.; Kemp, T. J. J. Phys. Chem. 1972, 76,20

(35) Burrows, H. D.; Castro, R. A. E.; Esteves, M. A.; Gigante, B.; Leitao, M. L. P.; Pauleta, A. C. Mater. Sci. Forum 2006, 504/506, 8.

(36) Burrows, H. D.; Seixas de Melo, J.; Forster, M.; Guntner, R.; Scherf, U.; Monkman, A. P.; Navaratnam, S. Chem. Phys. Lett. 2004, 385, 105.

(37) te Velde, G.; Bickelhaupt, F. M.; Baerends, E. J.; Guerra, C. F.; van Gisbergen, S. J. A.; Snijders, J. G.; Ziegler, T. J. Comput. Chem. 2001, 22, 931

(38) Vosko, S. H.; Wilk, L.; Nusair, M. Can. J. Phys. 1980, 58, 1200.

(39) Jensen, F. Introduction to Computational Chemistry; John Wiley \& Sons Ltd.: Chichester, England, 1999.

(40) Becke, A. D. Phys. Rev. A 1988, 38, 3098.

(41) Perdew, J. P.; Yang, W. Phys. Rev. B 1986, 33, 8800.

(42) Runge, E.; Gross, E. K. U. Phys. Rev. Lett. 1984, 52, 997.

(43) Petersilka, M.; Gossmann, U. J.; Gross, E. K. U. Phys. Rev. Lett. 1996, 76, 1212.

(44) Kong, J.; White, C. A.; Krylov, A. I.; Sherrill, D.; Adamson, R. D.; Furlani, T. R.; Lee, M. S.; Lee, A. M.; Gwaltney, S. R.; Adams, T. R.; Ochsenfeld, C.; Gilbert, A. T. B.; Kedziora, G. S.; Rassolov, V. A.; Maurice, D. R.; Nair, N.; Shao, Y.; Besley, N. A.; Maslen, P. E.; Korambath, J. P.; Baker, J.; Byrd, E. F. C.; van Voorhis, T.; Oumi, M.; Hirata, S.; Hsu, C.; Ishikawa, N.; Florian, J.; Warshel, A.; Johnson, B. G.; Gill, P. M. W.; HeadGordon, M.; Pople, J. A. J. Comput. Chem. 2000, 21, 1532.

(45) Dunning, T. H. J. Chem. Phys. 1989, 90, 1007.

(46) Lee, C.; Yang, W.; Parr, R. G. Phys. Rev. B 1988, 37, 785.

(47) Cornil, J.; Belijonne, D.; Bredas, J. L. J. Chem. Phys. 1995, 103 , 842.

(48) Grozema, F. C.; Siebbeles, L. D. A.; Warman, J. M.; Seki, S.; Tagawa, S.; Scherf, U. Adv. Mater. 2002, 14, 228.

(49) Ruscic, B.; Kovac, B.; Klasinc, L.; Gusten, H. Z. Naturforsch., A 1978, 33, 1006.

(50) Nemeth, G. I.; Selzle, H. L.; Schlag, E. W. Chem. Phys. Lett. 1993, 215,151 .

(51) Fratiloiu, S.; Candeias, L. P.; Grozema, F. C.; Wildeman, J.; Siebbeles, L. D. A. J. Phys. Chem. B 2004, 108, 19967.

(52) Fratiloiu, S.; Grozema, F. C.; Siebbeles, L. D. A. J. Phys. Chem. $B$ 2005, 109, 5644.

(53) Fesser, K.; Bishop, A. R.; Campbell, D. K. Phys. Rev. B 1983, 27, 4804.

(54) Grozema, F. C.; Candeias, L. P.; Swart, M.; van Duijnen, P. T.; Wildeman, J.; Hadziiannou, G.; Siebbeles, L. D. A.; Warman, J. L. J. Chem. Phys. 2002, 117, 11366. 